\title{
Economic Designing of Modified Chain Sampling Plan under Weibull Distribution using Bayesian Approach
}

\author{
P. Jeyadurga, S. Balamurali
}

\begin{abstract}
The methodology to design modified chain sampling plan with economical aspect for assuring product's mean life under Weibull distribution is considered in this paper. Bayesian approach is employed to estimate the unknown failure probability

involved in economic designing. The ratio between product's true mean life and specified mean life is assumed to be product's quality. The optimal parameters are chosen so that they simultaneously satisfy the risks of both producer and consumer with minimum cost using the optimization problem. In addition, different shape parameters are used in optimal parameters determination. Real life data is used to explain the execution of the proposed plan and the performance of the proposed plan is compared with other existing plan using average outgoing quality

curve.
\end{abstract}

Keywords: Bayesian approach, economic design, lifetime ratio, modified chain sampling, truncated life test..

\section{INTRODUCTION}

Statistical quality control (SQC) is referred to as the use of statistical methods in monitoring, maintaining and ensuring the quality of products. Control chart and acceptance sampling are two powerful techniques of SQC used to inspect the product's quality. Control chart tool is generally used to monitor the status of the production process and to maintain the process with consistency. In addition, process variation causes are analyzed and detected by using the control chart. However, control chart plays a vital role in maintaining the process at specified target level, it does not help to ensure the product quality after the production is completed whereas quality assurance is necessary. In this situation, another tool of SQC namely, acceptance sampling can be performed to provide the quality assurance of the product. Acceptance sampling involves the inspection of certain number of sample items which is randomly drawn from the submitted lot. The entire lot is accepted if the result obtained from the sample items satisfies the specified requirements otherwise it is

Revised Manuscript Received on December 15, 2019

* Correspondence Author

P. Jeyadurga, Department of Mathematicss, Kalasalingam Academy of Research and Education, Krishnankoil - 626126, Tamil Nadu, India. Email: jeyadurga.2810@yahoo.com.

S. Balamurali*, Department of Computer Applications, Kalasalingam Academy of Research and Education, Krishnankoil - 626126, Tamil Nadu, India.Email: sbmurali@rediffmail.com.

rejected. As the decision on the entire lot depends on the result of sample items and which are just a small quantity in the entire lot, there may be a chance to reject a qualified lot or to accept an unqualified lot. The risks associated with qualified lot's rejection and unqualified lot's acceptance are defined as producer's risk $(\alpha)$ and consumer's risk $(\beta)$ respectively. Acceptance sampling plans specify the sampling rules in order to make a decision on the lot. An operating characteristic (OC) curve graphically depicts the possibility of accepting the lot for different values of fraction non-conforming under a certain sampling plan. That is, the OC curve measures the performance of acceptance sampling plans and it depicts the discrimination of sampling plans between good and poor quality lots. In addition, sample size and acceptance number interact each other in determining the discrimination of the sampling plans. Zero acceptance number single sampling plan (SSP) requires large sample size to dispose the lot. The discriminating power of such plans is poor and also the $\mathrm{OC}$ curve has no inflection point. To solve these shortcomings of zero acceptance number SSP, chain sampling plan (ChSP-1) was introduced by [1]. In this ChSP-1 procedure, one failure item is allowed to current lot acceptance provided there are no failure items in preceding ' $i$ ' samples. Therefore, the OC curve of ChSP-1 has better shape

compared to the same of SSP with zero acceptance number. In

the literature, several authors studied ChSP-1 and its different

forms (see for example [2], [3], [4], [5]). Also, the sample size required for current lot disposition is reduced in ChSP-1 as it utilizes the preceding lots sample results. However, the achievement of sample size reduction under ChSP-1 is minimum. Hence, the modified procedure of ChSP-1 with the name modified ChSP-1 was proposed by [6]. This plan is often designated as MChSP-1 and improves the OC curve shape with minimum sample size rather than ChSP-1. Recently, few of the researchers designed this MChSP-1 for lot inspection using attribute and variables inspection (see for example, [7] and [8]).

In recent years, product's lifetime is treated as the major quality characteristic among others and also to obtain the failure time of the products, the lifetime experiment is conducted and truncated at pre-fixed time. In this test, the sample item is classified as

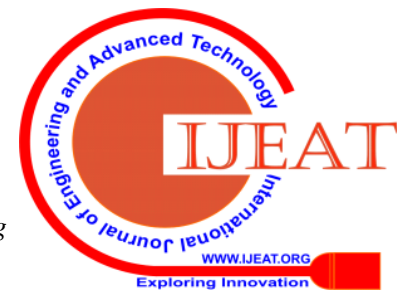


conforming if its functioning is not failed before the testing time. Otherwise, it is classified as non-conforming item. Many researchers designed the sampling plans based on time truncated life test for the purpose of providing product's lifetime assurance (see for example, [9], [10]).

Nowadays, the sampling plan designing with economic aspect attracts the researchers attention. In addition, the economic sampling plans will be admirable because it involves the finding of minimum cost to satisfy both producer and consumer risks. Several authors considered the economic designing of various sampling plans to reduce the inspection cost (see for example, [11], [12], [13]).

To the best of our knowledge, the economic designing of MChSP-1 using Bayesian approach to provide product's mean life assurance is not available in the literature. Therefore, this study attempts to construct the tables for optimal parameters of MChSP-1 to ensure product's mean life with minimum cost under Weibull distribution. The organization of the remaining paper is as follows. An introduction about the Weibull distribution employed under this study is provided in Section 2. The operating procedure and some of the performance measures of proposed MChSP-1 are derived using Bayesian approach and are given in Section 3. The designing approach as well as the execution of the proposed plan is discussed in Section 4. In Section 5, the comparative study on the performance of proposed plan and ChSP-1 is given using average outgoing quality. Some conclusions are provided in the last section.

\section{WEIBULL DISTRIBUTION}

Weibull distribution is considered as a suitable lifetime model to describe the observed failures of components or systems and this distribution is also widely used in different fields (see for example, [14], [15], [16]). Also, the Weibull distribution has been used in field of SQC for constructing the sampling plans and the literature consists of numerous studies on designing of sampling plans under this distribution (see for example, [17], [18]). In this study, the economic designing of

MChSP-1 using Bayesian approach is discussed to assure the product's mean life under Weibull distribution. The following

equation is known as the cumulative distribution function of the Weibull distribution.

$F(t ; \lambda, \delta)=1-\exp \left(-\left(\frac{t}{\lambda}\right)^{\delta}\right), t \geq 0, \lambda>0, \delta>0$

where $\delta$ and $\lambda$ are the respective shape and scale parameters. In this study, we assume that $\delta$ is known and $\lambda$ is unknown. In practice, the estimation of the shape parameter is possible by using the past history of production process. The probability that the product will fail before the experiment time $t_{0}$ under the Weibull distribution is given as

$$
p=1-\exp \left(-\left(\frac{\mathrm{t}_{0}}{\lambda}\right)^{\delta}\right)
$$

The Weibull distributed mean life of a product under is obtained by

$$
\mu=\left(\frac{\lambda}{\delta}\right) \Gamma\left(\frac{1}{\delta}\right)
$$

where $Г($.$) represents the complete gamma function. In$ general, when designing the sampling plans for providing lifetime assurance, the test duration $t_{0}$ is often expressed as a constant multiple of specified mean life $\mu_{0}$. In symbol, $t_{0}=a \mu_{0}$, where the constant ' $a$ ' is called as an experiment termination ratio. In this study, we also use such procedure to design the sampling plan. After the substitution of $\mu$ and $t_{0}$, equation (2) can be rewritten as follows

$$
p=1-\exp \left(-a^{\delta}\left(\frac{\mu_{0}}{\mu}\right)^{\delta}\left(\frac{\Gamma(1 / \delta)}{\delta}\right)^{\delta}\right)
$$

The failure probability of the product is obtained using the above equation when ensuring the Weibull distributed mean life. We can find the failure probabilities using the above equation if any values of mean ratio and shape parameter are specified.

\section{MCHSP-1 USING TRUNCATED LIFE TEST}

Obviously, MChSP-1 is implemented to make a decision on the submitted lots of high quality and which uses minimum sample size for lot sentencing purpose rather than ChSP-1. There is a difference between the operating procedure of MChSP-1 and ChSP-1. Specifically, in ChSP-1 the acceptance of preceding ' $i$ ' lots is considered only if current lot sample contains one failed item but the acceptance of preceding ' $i$ ' lots is always used in the current lot disposition under MChSP-1. Based on time truncated life test, the operating procedure of the proposed MChSP-1 is described as follows

1) Draw a sample of $n$ items randomly from the current lot and conduct the life test on the sample items for the specified time $t_{0}$. Observe and count number of sample items failed before the time $t_{0}$.

2) If current lot sample does not contain failed item and there is at most one failed item is found in preceding ' $i$ ' lots, then accept the current lot. Otherwise, reject the current lot.

MChSP-1 is characterized by two parameters namely sample size, $n$ and the number of preceding lots used in current lot disposition, $i$. The flow chart given in Fig. 1 reveals the operating procedure of MChSP-1 on the basis of time truncated life test.

The operating procedure of proposed MChSP-1 shows that the current lot will be accepted if current lot sample does not contain failed item as well as

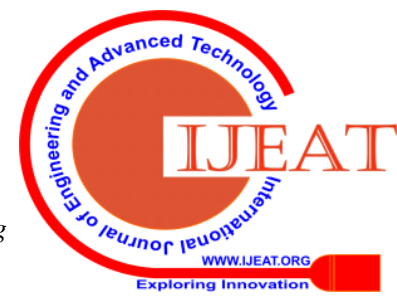


previous ' $i$ ' lot samples or there is at most one failed item is found in overall preceding ' $i$ ' samples. From this we can understand that the proposed plan will be suitable for the inspection of the products to ensure the best quality. Then the OC function of MChSP-1 is obtained by (see [6])

$$
P_{a}(p)=P_{0}\left\lfloor P_{0}^{i}+i P_{0}^{i-1} P_{1}\right\rfloor
$$

where $P_{a}(p)$ denotes the probability of acceptance of lot under MChSP-1 at given failure probability $p, P_{0}$ and $P_{1}$ are respective probabilities for the lot containing none and one failed item.

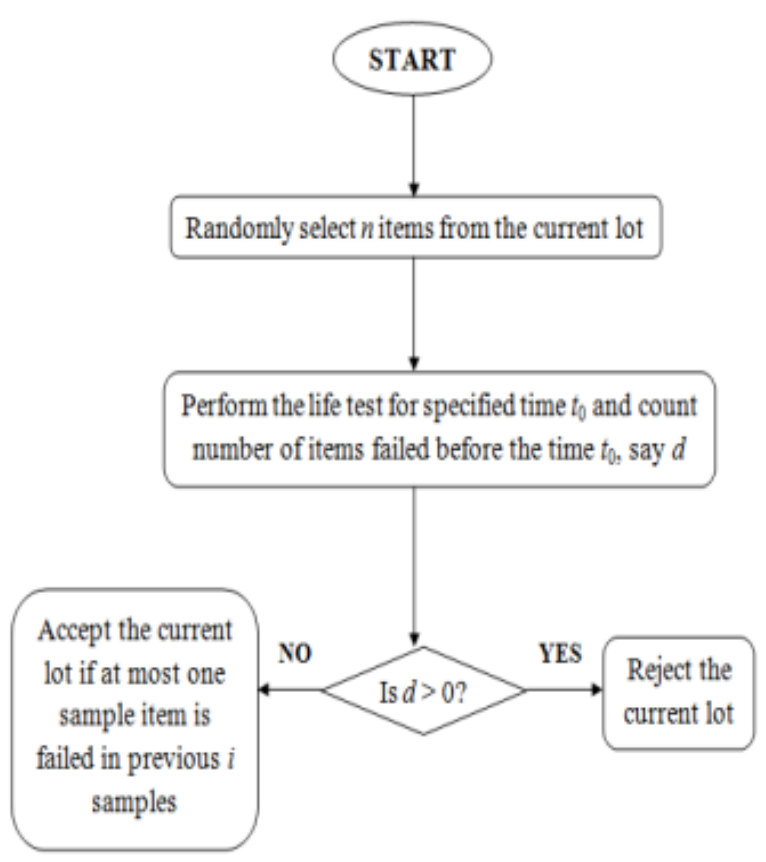

Fig. 1. Operating procedure of the MChSP-1

Using the binomial distribution, the OC function can be rewritten as follows

$$
P_{a}(p)=(1-p)^{n+n i-1}[(1-p)+i n p]
$$

where $n$ represents the number of sampled items and $i$ denotes

the number of preceding lots used for current lot disposition. In this study, the failure probabilities obtained by the mean ratios $4,6,8$ and 10 are considered as acceptable quality level (AQL) (or $p_{1}$ ) and similarly the same corresponding to the mean 1 is called as limiting quality level (LQL) (or $p_{2}$ ).

$$
\begin{aligned}
& P_{a}\left(p_{1}\right)=\left(1-p_{1}\right)^{n+n i-1}\left[\left(1-p_{1}\right)+i n p_{1}\right] \\
& P_{a}\left(p_{2}\right)=\left(1-p_{2}\right)^{n+n i-1}\left[\left(1-p_{2}\right)+i n p_{2}\right]
\end{aligned}
$$

The above equations (7) and (8) can be used to find the probabilities of acceptance of the lot at various AQL and LQL respectively.

\section{ECONOMIC DESIGN OF MCHSP-1 USING BAYESIAN APPROACH}

As mentioned earlier, economic designing of sampling plans plays an important role in the literature. Also, different approaches have been proposed to design the sampling plans with economic aspect (see for example, [19], [20]). The proposed study considers the economic designing of MChSP-1 for ensuring product's mean life with minimum cost. For this economic designing, we need some of the performance measures of the proposed plan namely, average total inspection (ATI) and average outgoing quality (AOQ) and are obtained by the following equations. $A T I=n+\left(1-P_{a}(p)\right)(N-n)$

$A O Q=\frac{p P_{a}(p)(N-n)}{N}$

where the lot acceptance probability under MChSP-1 is given in (6). Detection and non-detection of failure items are respectively denoted by $D_{d}$ and $D_{n}$ which are as follows $D_{d}=n p+\left(1-P_{a}(p)\right)(N-n) p$

$D_{n}=p P_{a}(p)(N-n)$

We define the cost parameters to obtain the total cost (TC) per lot under given sampling plan as $C_{i}=$ Life testing cost per item; $C_{f}=$ Cost of replacement; $C_{o}=$ Cost of an outgoing failure item.

In general, the value of $p$ involved in equations $(9-12)$ is unknown. The economic designing of SSP for specified values of $p$ was proposed by [11]. The group acceptance sampling plan designing with economic aspect for assuring Weibull distributed median life of the product has been studied by [12] in which the Bayesian approach has been used for the estimation of $p$ value. In proposed MChSP-1 designing, we use the Bayesian approach as used in [12] to determine the value of $p$. We assume that the prior distribution of $p$ follows the beta distribution with shape parameters $v>0$ and $\tau>0$. The beta distribution has the following probability density function.

$$
\operatorname{beta}(p)=\frac{p^{v-1}(1-p)^{\tau-1}}{B(v, \tau)}
$$

Using the information of $p$ follows beta distribution, the derivation for $A T I, D_{d}, D_{n}$ and $A O Q$ are done. For any combinations of $v$ and $\tau$, we can design the proposed plan using such derivation. But in this paper, we consider the values $v=1$ and $\tau=2$. Based on these specified values, the measures are obtained as follows $A T I=n+(N-n)\left[1-\frac{2(n+1)}{V}\right]$

$D_{d}=\frac{n}{3}+(N-n)\left[\left(\frac{1}{3}\right)-\left(\frac{2(n-n i+1)}{W}\right)\right]$ 


$$
\begin{gathered}
D_{n}=(N-n)\left[\left(\frac{2(n+3 n i+1)}{W}\right)\right] \\
A O Q=\frac{(N-n)}{N}\left[\left(\frac{2(n+3 n i+1)}{W}\right)\right]
\end{gathered}
$$

where $V=(n+n i+1)(n+n i+2)$ and $W=(n+n i+1)(n+n i+2)(n+n i+3)$ The following mathematical model is used to find the optimal parameters with minimum TC.

Minimize $\quad T C=C_{i} \cdot A T I+C_{f} \cdot D_{d}+C_{o} \cdot D_{n}$

Subject to $\quad P_{a}\left(p_{1}\right) \geq 1-\alpha$

$$
\begin{gathered}
P_{a}\left(p_{2}\right) \leq \beta \\
n>1, i \geq 1
\end{gathered}
$$

where $P_{a}\left(p_{1}\right)$ and $P_{a}\left(p_{2}\right)$ are probabilities of acceptance of lot at $\mathrm{AQL}$ and LQL respectively and obtained by using equations (7) and (8).

In order to determine the optimal parameters to assure the mean life of the product under Weibull distribution, one specified value of shape parameter is considered such as 2 .

The optimal parameters are determined so that with minimum cost the producer's risk and consumer's risk are satisfied simultaneously. The producer's risk is taken as = 0.05 and four values of consumer's risk are assumed as $\beta=$ $0.25,0.10,0.05,0.01$. The test termination ratio is considered as two cases namely $a=0.25$ and $a=0.5$. In addition, the set of specified input values such as lot size $N$ $=1000$ and $C_{i}=1.0 ; C_{f}=2.0 ; C_{o}=10$ is considered to determine the minimum TC along with ATI, $D_{d}, D_{n}$. The values of AOQ are not presented in the tables because it is just a $(1 / N)^{\text {th }}$ multiplication of $D_{n}$. Optimal values of $n, i$, $A T I, D_{d}, D_{n}$ and TC to assure product's mean life under Weibull distribution with shape parameter 2 for $a=0.25$ and $a=0.5$ are reported in Tables I and II. From these tables, some general trends are observed as follows.

(i) For fixed values of $\delta, a, \mu / \mu_{0}$, the values ATI, TC increase and $D_{n}$ decreases when $\beta$ decreases. There is no standard trend in the value of $D_{d}$. That is, $D_{d}$ may decrease or equal or increase. For example, if $\delta=2, a=0.25$ and $\mu / \mu_{0}=6$, $D_{d}=333.33$ when $\beta=0.10, D_{d}=333.41$ when $\beta=0.05$, $D_{d}=333.39$ when $\beta=0.01$.

(ii) When $\delta=2$ and $\mu / \mu_{0}=4$, the sample size decreases and $i$ increases if consumer's risk decreases from 0.25 to 0.10 . Similarly, when $\mu / \mu_{0}=6$, the sample size increases if consumer's risk decreases from 0.25 to 0.10 . But for the same mean ratio, sample size is decreased and $i$ increases if there is decrement in $\beta$ from 0.10 to 0.05 (or 0.01 ). In all the cases, the optimal values corresponding to the mean ratios $\mu / \mu_{0}=8$ and 10 are same. The above trends are same either $a=0.25$ or $a=0.5$.
Table I Optimal parameters of economic MChSP-1 to ensure the Weibull distributed mean life when $\delta=2$ and $a=0.25$

\begin{tabular}{|c|c|c|c|c|c|c|c|}
\hline$\beta$ & $\mu / \mu_{0}$ & $n$ & $i$ & $A T I$ & $D_{d}$ & $D_{n}$ & $T C$ \\
\hline 0.25 & 4 & 13 & 3 & 990.34 & 333.65 & 1.64 & 1659.28 \\
& 6 & 22 & 1 & 978.27 & 333.31 & 1.79 & 1646.68 \\
& 8 & $\uparrow$ & $\uparrow$ & $\uparrow$ & $\uparrow$ & $\uparrow$ & $\uparrow$ \\
& 10 & $\uparrow$ & $\uparrow$ & $\uparrow$ & $\uparrow$ & $\uparrow$ & $\uparrow$ \\
& & & & & & & \\
0.10 & 4 & 10 & 7 & 996.72 & 333.55 & 0.79 & 1664.61 \\
& 6 & 34 & 1 & 986.00 & 333.33 & 0.77 & 1653.43 \\
& 8 & $\uparrow$ & $\uparrow$ & $\uparrow$ & $\uparrow$ & $\uparrow$ & $\uparrow$ \\
& 10 & $\uparrow$ & $\uparrow$ & $\uparrow$ & $\uparrow$ & $\uparrow$ & $\uparrow$ \\
& & & & & & & \\
0.05 & 4 & $*$ & $*$ & $*$ & $*$ & $*$ & $*$ \\
& 6 & 30 & 2 & 992.82 & 333.41 & 0.53 & 1660.15 \\
& 8 & 43 & 1 & 989.00 & 333.33 & 0.49 & 1656.15 \\
& 10 & $\uparrow$ & $\uparrow$ & $\uparrow$ & $\uparrow$ & $\uparrow$ & $\uparrow$ \\
& & & & & & & \\
& 4 & $*$ & $*$ & $*$ & $*$ & $*$ & $*$ \\
& 6 & 27 & 4 & 997.08 & 333.39 & 0.27 & 1664.13 \\
& 8 & 62 & 1 & 992.50 & 333.33 & 0.23 & 1659.39 \\
& 10 & $\uparrow$ & $\uparrow$ & $\uparrow$ & $\uparrow$ & $\uparrow$ & $\uparrow$ \\
\hline
\end{tabular}

* Plan does not exist.

$\uparrow$ : Use the plan above.

Table II Optimal parameters of economic MChSP-1 to ensure the Weibull distributed mean life when $\delta=2$ and $a=0.5$

\begin{tabular}{|c|c|c|c|c|c|c|c|}
\hline$\beta$ & $\begin{array}{c}\mu / \mu \\
0\end{array}$ & $n$ & $i$ & $A T I$ & $D_{d}$ & $D_{n}$ & $T C$ \\
\hline
\end{tabular}




\begin{tabular}{|c|c|c|c|c|c|c|c|}
\hline 0.2 & 4 & 3 & 4 & 970.6 & 336.5 & 16.2 & 1660.1 \\
\hline \multirow[t]{5}{*}{5} & 6 & 6 & 1 & 8 & 9 & 9 & 5 \\
\hline & 8 & $\uparrow$ & $\uparrow$ & 923.5 & 332.6 & 18.2 & 1606.9 \\
\hline & 10 & $\uparrow$ & $\uparrow$ & 4 & 1 & 1 & 5 \\
\hline & & & & $\uparrow$ & $\uparrow$ & $\uparrow$ & $\uparrow$ \\
\hline & 4 & 2 & 10 & $\uparrow$ & $\uparrow$ & $\uparrow$ & $\uparrow$ \\
\hline 0.1 & 6 & 9 & 1 & & & & \\
\hline \multirow[t]{5}{*}{0} & 8 & $\uparrow$ & $\uparrow$ & 989.1 & 335.7 & 9.11 & 1669.8 \\
\hline & 10 & $\uparrow$ & $\uparrow$ & 5 & 9 & 9.19 & 5 \\
\hline & & & & 947.8 & 333.0 & $\uparrow$ & 1623.2 \\
\hline & 4 & $*$ & $*$ & 4 & 9 & $\uparrow$ & 0 \\
\hline & 6 & 8 & 2 & $\uparrow$ & $\uparrow$ & & $\uparrow$ \\
\hline 0.0 & 8 & 11 & 1 & $\uparrow$ & $\uparrow$ & $*$ & $\uparrow$ \\
\hline \multirow[t]{5}{*}{5} & 10 & $\uparrow$ & $\uparrow$ & & & 6.44 & \\
\hline & & & & $*$ & $*$ & 6.45 & $*$ \\
\hline & 4 & $*$ & $*$ & 972.5 & 334.1 & $\uparrow$ & 1647.2 \\
\hline & 6 & 7 & 4 & 3 & 2 & & 2 \\
\hline & 8 & 16 & 1 & 957.0 & 333.1 & $*$ & 1629.8 \\
\hline 0.0 & 10 & $\uparrow$ & $\uparrow$ & 0 & 9 & 3.61 & 3 \\
\hline \multirow[t]{7}{*}{1} & & & & $\uparrow$ & $\uparrow$ & 3.26 & $\uparrow$ \\
\hline & & & & $*$ & $*$ & & $*$ \\
\hline & & & & 988.0 & 334.1 & & 1659.9 \\
\hline & & & & 7 & 2 & & 2 \\
\hline & & & & 970.1 & 333.2 & & 1640.0 \\
\hline & & & & 8 & 8 & & 1 \\
\hline & & & & $\uparrow$ & $\uparrow$ & & $\uparrow$ \\
\hline
\end{tabular}

* Plan does not exist.

$\uparrow$ : Use the plan above.

\section{ILLUSTRATIVE EXAMPLE}

Suppose that the manufacturer of ball bearing wants to test the reliability of his product. He specifies that the average life of the product is 0.200 . That is, $\mu_{0}=0.200$. It is assumed that the lifetime follows Weibull distribution with shape parameter $\delta=2$. The producer's risk and consumer's risk are considered to be $\alpha=0.05$ and $\beta=0.10$ when $\mu / \mu_{0}=6$. The test duration is taken as $t_{0}=0.100$. Hence, it is calculated that the experiment termination ratio is $a=0.5$. For this testing purpose, it is decided to implement the proposed economic MChSP-1 and the optimal parameters of the proposed plan are obtained from Table I for above specified requirements such as $n=9, i=1$. For the proposed plan implementation, a real time data of compressive strength of ball bearings is considered as discussed in [17]. There is compressive strength of 21 ball bearings available and it is considered as the lifetime of the ball bearings. We consider the first 18 data for our illustration purpose since here the sample size is $n=9$ and $i=1$. That is, we need the lifetime data for current lot the lifetime of the sample items obtained from the current lot is as follows.
0.5582
0.2439
0.4728
0.1764
0.1239
0.8787
0.3995
0.1687
0.3858

We can observe from the above data that there is none of the ball bearing failed before 0.100 . We consider the lifetime data of preceding lot sample ball bearings because $i=1$. sample size as well as preceding lot sample size. Suppose that

Suppose that the lifetime of ball bearings obtained from preceding lot is as follows.
0.4427
$0.1050 \quad 0.4447$
0.3119
0.3733
0.7539
0.1624
$0.5920 \quad 0.8900$

There is no ball bearing of preceding lot failed before the experiment time is reached. Hence, the current lot is accepted under the proposed plan.

\section{COMPARATIVE STUDY}

As the proposed plan considers preceding ' $i$ ' lots sample information for current lot disposition even there is none of the sample item failed, the inspection under MChSP-1 will be very stringent. But when implementing the ChSP-1 it uses preceding samples information if one failed item is found in current lot sample items. From this we can understand that the AOQ after implementation of the proposed plan is smaller than the AOQ of ChSP-1. Therefore, we compare the AOQ of both MChSP-1 and ChSP-1 using the AOQ curves. To draw the AOQ curves, we select an optimal sampling plan so that which simultaneously satisfy the producer's risk of 0.05 and consumer's risk of 0.25 when $a=0.25$ and $\mu / \mu_{0}=4$ and also this plan is determined to ensure Weibull distributed mean life of the products when shape parameter $\delta=2$. The optimal parameters of proposed MChSP-1 are $n=13, i=3$ and the same for ChSP-1 are $n=30, i=2$. The AOQ curves corresponding to the above mentioned plans are shown in Fig. 2. From this figure, it can be observed that the AOQ of both plans are coincident up to certain stage when the values of failure probability are small. But there is large difference between the AOQ of ChSP-1 and MChSP-1 when there is an increment in failure probability. This represents that the non-detection of failure items under ChSP-1 is higher than that of MChSP-1. From this, we can understand that the proposed plan will provide more protection to the consumer rather than ChSP-1. Consequence of high protection of consumer, the producer will achieve good reputation for his products. Therefore, we conclude from this discussion that the proposed MChSP-1 will be favorable to both producer and consumer.

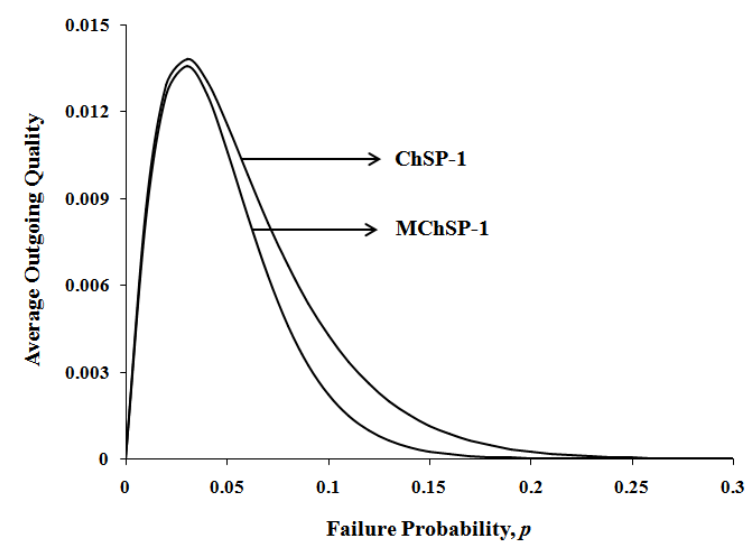

Fig. 2. AOQ curves of proposed MChSP-1 and ChSP-1 


\section{CONCLUSIONS}

As lifetime is one of the important quality characteristics of the product, we have proposed an economic designing of MChSP-1 using Bayesian approach under Weibull distribution for ensuring mean life of the products. The flow chart has been given to explain the operating procedure of the proposed plan and we have provided the tables for the purpose of optimal parameters selection. The execution of the proposed plan has been discussed using the real life data and we have made a comparative study between the proposed plan and ChSP-1 using one of the performance measures namely AOQ. It has been concluded from the comparative study that the proposed plan will be efficient in protecting both producer and consumer.

\section{ACKNOWLEDGMENT}

The authors would like to thank the editor and anonymous reviewers for their precious suggestions and comments which significantly led to improve the presentation of the manuscript. The first author would like to thank the Kalasalingam Academy of Research and Education for providing financial support through Post-doctoral fellowship.

\section{REFERENCES}

1. H. F. Dodge, "Chain sampling inspection plan", Ind. Qual. Control, vol. 11, 1955, pp. 10-13.

2. K. Govindaraju, "Chain Sampling, Springer Handbook of Engineering Statistics”, London: Springer-Verlag, 2006, pp. 263-279.

3. R. Vijayaraghavan, and K. M. Sakthivel, "Chain sampling inspection plans based on Bayesian methodology", Econ. Qual. Control, vol. 26, 2011, pp. 173-187.

4. S. Balamurali, and M. Usha, "Optimal designing of variables chain sampling plan by minimizing the average sample number", Int. J. Manuf. Eng., Article ID 751807, 2013, pp. 1-12.

5. S. Balamurali, P. Jeyadurga, M. Usha, and M. Venkatesulu, "Optimal designing of chain sampling plan for Weibull distributed percentile life assurance", Int. J. Math. Comput., vol. 29, 2018, pp. 94-105.

6. K. Govindaraju, and C. D. Lai, "A modified ChSP-1 chain sampling plan, MChSP-1, with very small sample sizes", Amer. J. Math. Manage. Sci., vol. 8, 1998, pp. 343-358.

7. S. Balamurali, and M. Usha, "Designing of variables modified chain sampling plan based on the process capability index with unknown mean and variance", Amer. J. Math. Manage. Sci., vol. 36, 2017, pp. 363-377.

8. S. Luca, "Modified chain sampling plans for lot inspection by variables and attributes", J. Appl. Stat., vol. 45, 2018, pp. 1447-1464.

9. G. S. Rao, K. Rosaiah, M. S. Babu, and D. C. Sivakumar, "New acceptance sampling plans based on percentiles for exponentiated Frechet distribution", Econ. Qual. Control, vol. 31, 2016, pp. 37-44.

10. W. Gui, and M. Aslam, "Acceptance sampling plans based on truncated life tests for weighted exponential distribution”, Commun. Stat. Simul. Comput., vol. 46, 2017, pp. 2138-2151.

11. L. F. Hsu, and J. T. Hsu, "Economic design of acceptance sampling plans in a two-stage supply chain", Adv. Decis. Sci., Article ID 359082, 2012, pp. 1-14.

12. M. Aslam, M. Azam, S. Balamurali, and C. -H. Jun, “An economic design of a group sampling plan for a Weibull distribution using a Bayesian approach", J. Test. Eval., vol. 43, 2015, pp. 1497-1503.

13. M. S. Fallahnezhad, T. JafariNodoushan, M. S. Owlia, and M. H. Abooie, "Designing an economic repetitive sampling plan in the presence of two markets", IJE Trans. A: Basics, vol. 30, 2017, pp. 1017-1028.

14. D. Fabiani, and L. Simoni, "Discussion on application of the Weibull distribution to electrical breakdown of insulating materials", IEEE Dielect. Elect. Insul., vol. 12, 2005, pp. 11-16.
15. S. B. Li, and H. J. Lu, "Application of Weibull distribution in calculating ground deformation”, Appl. Mech. Mater., vol. 256-259, 2013, pp. 15-18.

16. M. H. Baqerin, Y. Shafahi, and H. Kashani, "Application of Weibull analysis to evaluate an forecast schedule performance in repetitive projects", J. Constr. Eng. Manage., vol. 142, 2016, pp. 1-9.

17. M. Aslam, M. Azam, and C. -H. Jun, "Acceptance sampling plans for multi-stage process based on time-truncated test for Weibull distribution", The Int. J. Adv. Manuf. Tech., vol. 79, 2015, pp. 1779-1785.

18. S. Balamurali, P. Jeyadurga, and M. Usha, "Optimal designing of multiple deferred state sampling plan for assuring percentile life under Weibull distribution”, The Int. J. Adv. Manuf. Tech., vol. 93, 2017, pp. 3095-3109.

19. W. G. Ferrell, and Jr. A. Chhoker, "Design of economically optimal acceptance sampling plans with inspection error", Comput. Oper. Res., vol. 29,2002, pp. 1283-1300.

20. M. S. Fallahnezhad, and A. Ahmadi Yazdi, "An optimization model for economic design of sampling plans based on conforming run length considering outgoing quality", Commun. Stat. Theor. Methods, vol. 46, 2017, pp. 2202-2211.

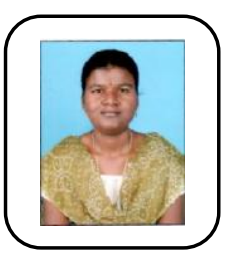

\section{AUTHORS PROFILE}

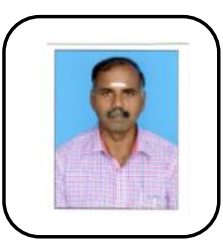

S. Balamurali is a professor of Statistics at Kalasalingam University. He earned his M. Sc. and Ph. D. degrees from Bharathiyar University, India. His research interests include statistical process control, acceptance sampling and analysis of means.

P. Jeyadurga completed her M. Sc. in Mathematics in Madurai Kamaraj University and received her Ph. D., degree from Kalasalingam Academy of Research and Education. Her areas of interest are acceptance sampling, control charts. 